\title{
A CS and SFCW Based Reconstruction Algorithm for Through-the-Wall Radar Imaging
}

\author{
Sun Yanpeng ${ }^{1,2}$, Cui Zhao ${ }^{1}$, Qu Lele ${ }^{1}$ \\ ${ }^{1}$ Department of Electronic Information Engineering, Shenyang Aerospace University, Shenyang, China \\ ${ }^{2}$ College of Information Science and Engineering, Northeastern University, Shenyang, China
}

Email address:

907323757@qq.com (Sun Yangpeng),polopolo88@126.com (Cui Zhao)

\section{To cite this article:}

Sun Yanpeng, Cui Zhao, Qu Lele. A CS and SFCW Based Reconstruction Algorithm for Through-the-Wall Radar Imaging. Journal of Electrical and Electronic Engineering. Vol. 3, No. 5, 2015, pp. 165-169. doi: 10.11648/j.jeee.20150305.21

\begin{abstract}
To satisfy with the increasingly exacting demand for real-time and high resolution requirements of TWRI, a Compressive Sensing (CS) based TWRI algorithm is proposed after the exploitation of Stepped-Frequency-Continuous-Wave (SFCW) system and signal's sparsity. Contrasted with the traditional imaging methods, this algorithm achieved precise targets localization and low sidelobe results with less computational time. The validity of the proposed CS imaging method is verified by simulation.
\end{abstract}

Keywords: TWRI, SFCW, Sparsity, Compressive Sensing

\section{基于压缩感知和频率步进连续波体制的穿墙雷达成像算法}

\author{
孙延鹏 ${ }^{1,2}$, 崔昭 ${ }^{1}$, 屈乐乐 ${ }^{1}$
}

沈阳航空航天大学, 电子信息工程学院, 沈阳, 辽宁, 中国

${ }^{2}$ 东北大学, 信息科学与工程学院, 沈阳, 辽宁, 中国

\section{邮箱}

907323757@qq. com（孙延鹏）, polopolo88@126. com（崔昭）

\begin{abstract}
摘要: 为了满足穿墙雷达成像技术 (Through-the-Wall Radar Imaging, TWRI) 对目标精准定位与探测实时性的要求, 本文通过对频率步进连续波 (Stepped Frequency Continuous Wave, SFCW) 雷达体制和空间信号的稀疏性分析，提出 一种基于压缩感知 (Compressive Sensing, CS) 的成像算法。与传统穿墙雷达成像算法相比, 本算法在重建结果上具 有成像目标定位准确, 旁瓣收敛度高, 计算耗时较少等方面的优点。仿真结果证明了算法的可行性和优越性。
\end{abstract}

关键词: 穿墙雷达, 频率步进连续波, 稀疏性, 压缩感知

\section{1. 引言}

随着近几年的科技的进步和发展，穿墙雷达成像技术 (Through-the-Wall-Radar-Imaging, TWRI) [1] 已经成为当 今各种非破坏性探测成像方法中最具有应用前景的穿透 探测方法之一。超宽带雷达成像技术在实际工程中的开发 应用已经成为时下热议的话题, 在反恐反爆行动中的实时 监测, 刑侦案件中的环境勘测, 考古过程中的空间探测等
诸多领域, 超宽带雷达成像技术 [2] 都因其良好的非侵入 式探测能力而展现出无可比拟的优势。随着穿墙雷达成像 范围的扩大和分辨率要求的不断提高, 使得穿墙雷达系统 数据采集量大、数据采集周期长, 成像过程慢等缺点更加 突出。传统的成像算法如后向投影成像算法 [3] 等已经不 能满足探测的实时性和高分率的要求, 因此, 如何高效的 缩短系统的数据采集时间、提高成像速度与精度成为急需 解决的难点。 
近年，压缩感知理论 [4]作为一种新的信号处理理论 被提出且在信息处理以及相关领域造成巨大影响, 其核心 概念即对于稀疏信号可以通过远少于奈奎斯特采样定理 所采集的信息实现信号的高效重建，目前压缩感知理论在 诸多工程领域受到广泛关注, 为解决穿墙雷达成像过程中 上述问题提供了理论基础。

\section{2. 数据获取方法}

本文采用收发分置 [6]的雷达天线在沿平行于墙面且 距离为 $h$ 的水平界面上以均等步长移动。假设墙体厚度为 $d$, 天线移动中的每个扫描位置, 在与发射天线以相对固 定距离 $\Delta L$ 处设置接收天线用以接收经过目标反射后的 回波信号, 收发天线连续步进工作直到对探测区域完成探 测过程。如图1所示雷达工作过程。

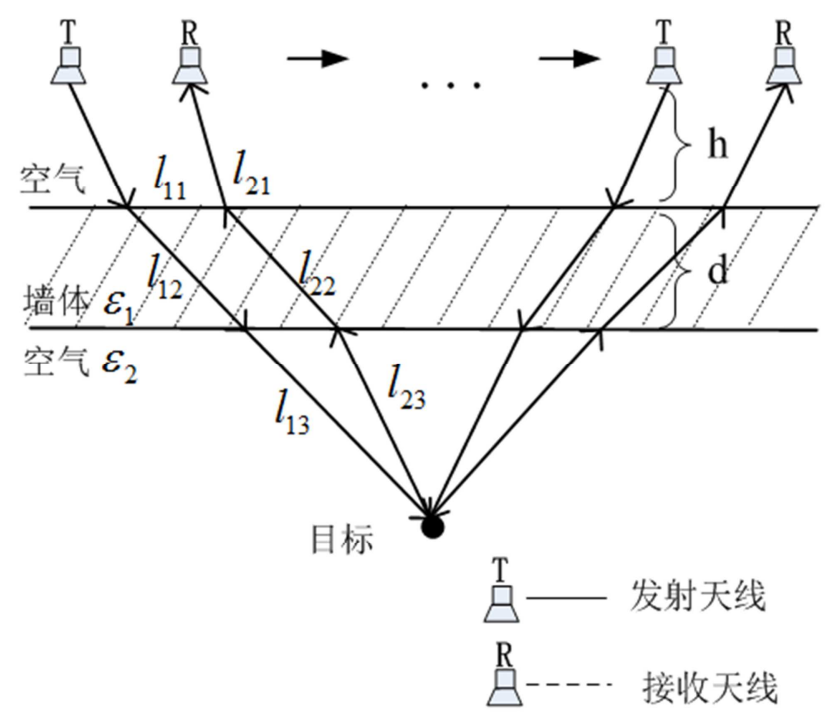

图1 雷达工作过程示意图。

在传统的SAR系统中, 频率步进连续波 [5]体制作为雷 达的工作体制之一, 采用以固定的频率间隔上升的电磁波 作为发射波, 在一个扫描周期内, 雷达发射的脉冲信号频 率从 $f_{0}$ 开始, 以频率间隔 $\Delta f$ 逐步上升, 直到最大扫描频 率 $f_{H}$ 。若雷达工作带宽 $B=f_{H}-f_{0}$, 每个天线扫描位置都 有 $M$ 个扫描频点, 那么频率间隔 $\Delta f=\left(f_{H}-f_{0}\right) / M$, 第 $m$ 个脉冲的频率如式 (1) 所示:

$$
f_{m}=f_{0}+m \Delta f, \quad m=0,1, \ldots, M-1
$$

空气和墙体的介电常数分别为 $\varepsilon_{1}$ 和 $\varepsilon_{2}, \sigma_{p}$ 表示第 $p$ 个点目标的复反射系数, $\tau_{p}$ 表示电磁波第 $n$ 对天线与墙后 第 $p$ 个点目标之间发射和接收过程的双路时延, 可通过公 式（2）计算得到:

$$
\tau_{p}=\left(l_{11}+l_{13}+l_{21}+l_{23}\right) / v_{1}+\left(l_{12}+l_{22}\right) / v_{2}
$$

其中, $v_{1}$ 和 $v_{2}$ 分别代表电磁波在空气和墙体中的传 播速度, $v_{1}$ 通常可以认为是 $3 \times 10^{8} \mathrm{~m} / \mathrm{s}, v_{2}$ 可以通过斯 奈尔定理（Snell's law） [7] 获得, 即 $v_{2}=v_{1} \sqrt{\varepsilon_{2} / \varepsilon_{1}}$ 。 每个测量孔径获取的目标回波信号的表达式可以表示为 式 (3):

$$
y(t)=\sum_{p=1}^{P} \sum_{m=0}^{M-1} \sigma_{p} \exp \left(-j 2 \pi f_{m} \tau_{p}\right)
$$

此处, $M$ 为在每一个测量孔径电磁波发射的频点数, 设 $T_{d}$ 每个频率持续的时间, $T_{s}$ 为天线发射信号的周期, 则 $T_{d}=T_{s} / M$ 。对获取到的回波数据进行初步处理, 利用 墙体和目标回波数据的相对差异性, 可以通过均值滤波法 [8]对墙体噪声进行滤除。

\section{3. 基于压缩感知的成像算法}

在每个测量孔径对应的扫描周期中, 频率从 $f_{0}$ 到 $f_{H}$ 共 $M$ 个频点, 测量孔径共 $N$ 个, 所以接收端的回波数据 可以表示为由 $N$ 个列向量组成, 其表示形式如式 (4) 所 示:

$$
\mathbf{y}=\left[\mathbf{y}_{1}, \mathbf{y}_{2}, \ldots, \mathbf{y}_{n}, \ldots, \mathbf{y}_{N-1}\right]^{T}
$$

其中任意一个列向量可表示为式（5）：

$$
\mathbf{y}_{n}=\left[\mathrm{y}_{n}\left(f_{0}\right), \ldots, \mathrm{y}_{n}\left(f_{m}\right), \ldots, \mathrm{y}_{n}\left(f_{M-1}\right)\right]^{T}
$$

压缩感知的核心理念是通过少量的观测值来恢复原 始信号, 将经过均值滤波后的原始回波数据 $\mathbf{y}$ 按列堆叠为 一个 $M N \times 1$ 维的列向量, 并通过一个非相关的采样矩阵 $\boldsymbol{\Phi}\left(\boldsymbol{\Phi} \in C^{J \times M N}, J \ll M N\right)$ 投影到低维的观测信号 $\mathbf{x}$ 上, 以达到压缩采样 [9] 的目的。则观测信号 $\mathbf{x}$ 与 $\mathbf{y}$ 的关系可 以表示为如式（6）所示:

$$
\mathbf{x}=\boldsymbol{\Phi} \mathbf{y}=\boldsymbol{\Phi} \Psi \mathbf{r}+\mathbf{n}=\boldsymbol{\Theta r}+\mathbf{n}
$$

其中, $\boldsymbol{\Theta}=\boldsymbol{\Phi} \boldsymbol{\Psi}$, 若最终成像网格个数为 $K \times L, \mathbf{r}$ 表示 $K L \times 1$ 维系数向量, $\mathbf{n}$ 为加性高斯白噪声, $\boldsymbol{\Psi}$ 代表 对 $\mathbf{y}$ 离散化处理得到的 $M N \times K L$ 维字典矩阵, 可通过式 (7) 得到。

$$
\boldsymbol{\Psi}_{i p}=\exp \left(-j 2 \pi f_{m} \tau_{p}\right)
$$

式中, $m=i \bmod M, n=\lceil i / M\rceil$, 对每一个用来成像 的网格, 都有 $M \times N$ 个对应的过完备字典元素, 即 $i=0,1, \ldots, M N-1$ 。

通过求解 $l_{1}$ 范数的最优化问题, 可得到 $K L \times 1$ 维反射 系数列向量 $\mathbf{r}$ 的估值, 本文中通过OMP算法 [10]求解此凸 优化问题。求解过程如式 (8) 所示: 


$$
\min _{\mathbf{r}}\|\mathbf{r}\|_{l_{1}} \quad \text { s.t }\left\|\Theta^{T}(\mathbf{x}-\Theta \mathbf{r})\right\|_{\infty}<\mu
$$

其中 $\mu$ 值取决于噪声方差 $\sigma$ 和数据采集量 $\lambda$, 可由 $\mu=\sigma \sqrt{2 \times \lg (\lambda)}$ 得到, 将重建后得到的反射系数向量 $\mathbf{r}$ 转换为与成像网格数对应的 $K \times L$ 维矩阵, 最终实现图像 重建。

\section{4. 实验仿真及数据分析}

实验中分别通过BP成像算法和CS重建算法进行重建, 以仿真实验结果和数据统计分析对比突出 CS 重建算法的 优越性。首先建立二维仿真探测区域，在墙后空间内部设 置四个理想点目标，其对应反射系数为 0.3 , 收发天线间 距 $0.2 \mathrm{~m}$, 天线距墙面 $0.3 \mathrm{~m}$, 天线移动步长 $0.25 \mathrm{~m}$, 墙 体厚度为 $0.2 m$, 测量孔径数为 41 , 扫描探测场景如图 2 所示。系统工作带宽为 $2 G$, 系统起始工作频率为 $1 \mathrm{GHz}$, 终止频率为 $3 \mathrm{GHz}$, 频点数为 101 , 频率步进间隔为 $20 \mathrm{MHz}$, 当系统信噪比 (SNR) 为 $3 d B$ 时, 系统频率响应 如图3所示,

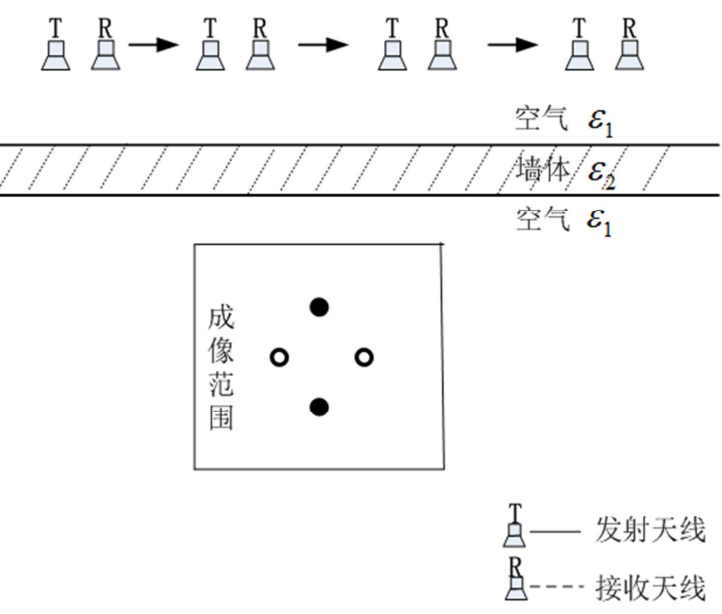

图2 雷达扫描探测场景。

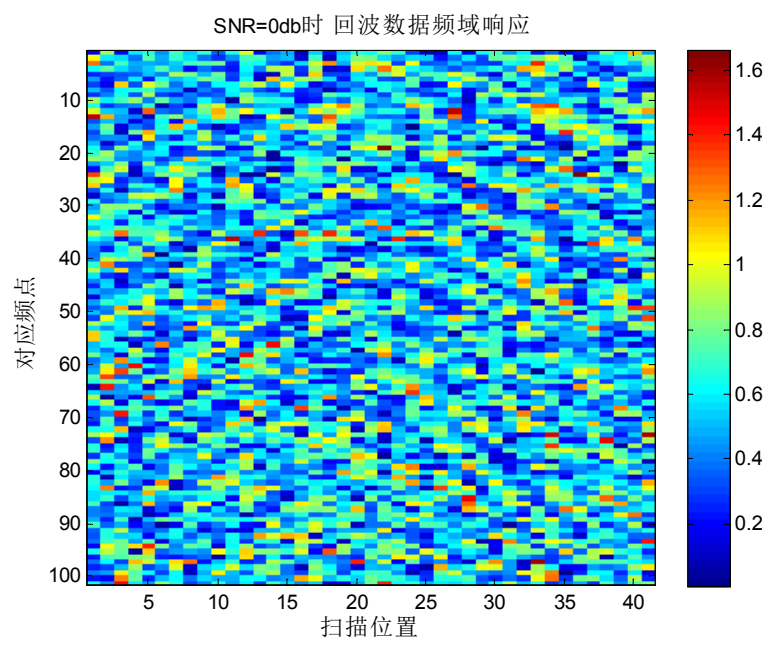

图3 系统频率响应。
如图2中成像范围所指, 将 $1.0 m \times 1.0 m$ 的墙后目标存 在区域作为成像空间并划分为 $41 \times 41$ 个成像网格。当回 波信噪比为 $3 d B$ 时, 分别通过两种成像算法进行成像。BP 成像算法重建过程采用均值滤波后的全部数据, 重建结果 如图4所示; 在基于CS的算法成像过程中, 对压缩采样后 的回波数据进行重建, 重建结果如图5所示。

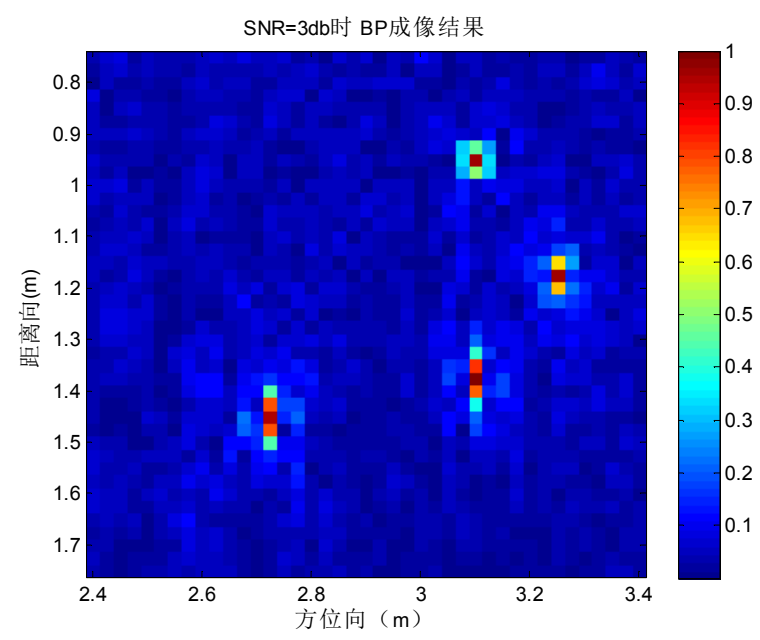

图4 $\mathrm{SNR}=3 \mathrm{~dB}$ 时 $\mathrm{BP}$ 算法成像。

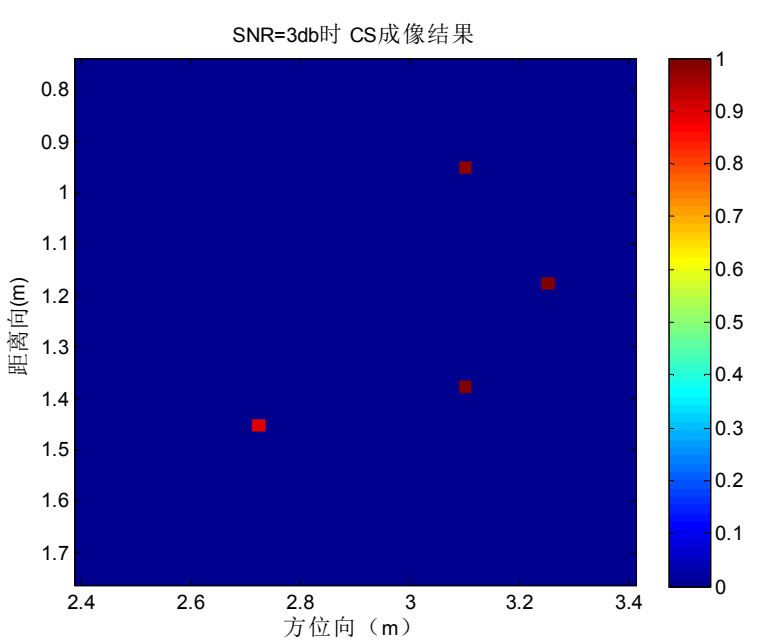

图5 $\mathrm{SNR}=3 \mathrm{~dB}$ 时基于 $\mathrm{CS}$ 的算法成像。

通过观察可以发现, 传统BP成像算法可以对墙后目标 进行良好的定位, 但是成像目标周围存在旁瓣较多, 受噪 声干扰严重等缺点, 相比之下, 基于CS的成像算法仍可以 清晰的分辨成像目标所在网格位置, 而且成像点目标周围 基本不存在旁瓣干扰, 随机噪声也得到了很好的抑制。

在考察验证成像点旁瓣收敛情况, 以及成像目标反射 系数重建真实性时, 通过对重建结果进行信杂比 (Target to Clutter Ratio, TCR ) [11] 和归一化均方误差 (Normalized Mean of Squared Error, NMSE) 的统计来 具体分析。其中重建信杂比的计算方法如公式 (9) 所示:

$$
T C R=10 \log 10\left(P / P^{\prime}\right)
$$


其中, $P$ 代表目标点对应多次实验重建的反射系数向 量内部真值的均值, $P^{\prime}$ 表示除目标点成像区域外杂波的 均值。对于归一化均方误差, 可以由公式 (10) 定义为:

$$
N M S E=\left\|\sigma-\sigma^{\prime}\right\|_{2} /\|\sigma\|_{2}
$$

$\sigma^{\prime}$ 和 $\sigma$ 分别代表重建反射系数和真实反射系数。此 两种成像算法在信噪比分别为 $5 d B$ 的探测环境中的重建 结果经归一化后成像, 以方便进行数据统计分析。两种成 像方法的重建结果具体表现如表1中所示:

表1 重建数据分析对比。

\begin{tabular}{lllll}
\hline 成像算法 & SNR & NMSE & TCR & 运行时间 \\
\hline BP成像算法 & $5 \mathrm{~dB}$ & $21.99 \%$ & $9.992 \mathrm{~dB}$ & $25.3152 \mathrm{~s}$ \\
CS成像算法 & $5 \mathrm{~dB}$ & $3.302 \%$ & $50.047 \mathrm{~dB}$ & $7.5731 \mathrm{~s}$ \\
\hline
\end{tabular}

通过两种成像算法分别在回波数据信噪比比值按 $-5 d B \sim 5 d B$ 连续变化的环境中进行重建, 在信噪比比值 不同的环境中分别进行数据采集并进行 40 次蒙特卡洛 (Monte-Carlo) 重建实验, 统计重建结果的变化趋势进行 对比, 如图6和图7所示: 通过观察分析, 可以看出基于CS 的成像算法在目标定位以及重建目标信息真实性上的表 现要远好于BP成像算法。

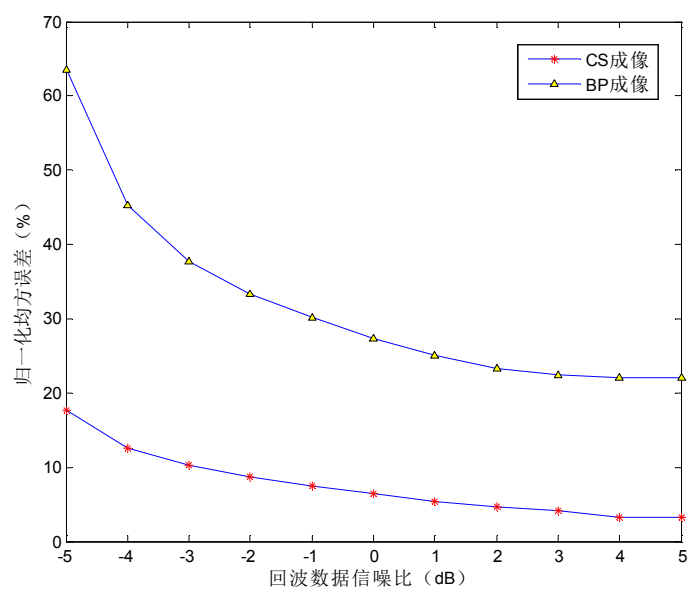

图6 重建归一化均方误差。

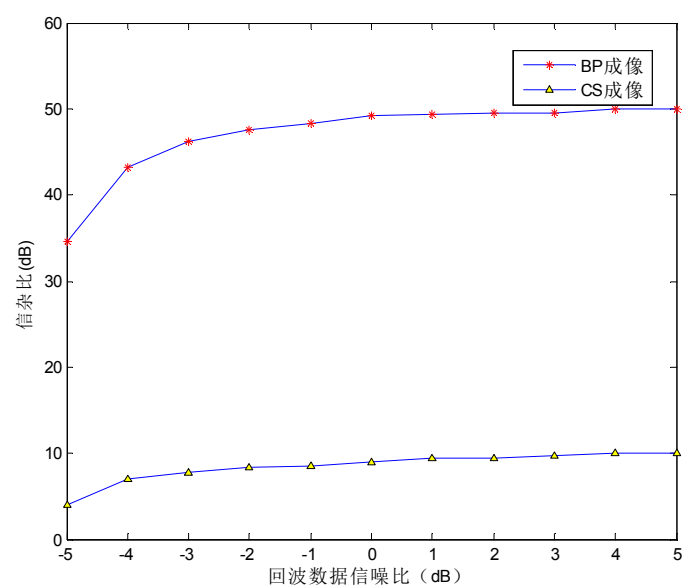

图7 重建信杂比。

\section{5. 结束语}

本文采用频率步进连续波雷达的工作体制，通过对信 号稀疏性研究分析, 提出一种压缩感知的成像算法, 对墙 后空间进行建模并用凸优化求解方式重建仿真试验场景。 仿真实验结果证明, 与传统穿墙雷达成像算法相比, 此算 法在大比例压缩减少实验数据的前提下, 高效重建了成像 区域信息, 具有成像目标定位准确, 旁瓣收玫度高, 计算 耗时较少等方面的优点。

\section{致谢}

本文为国家自然科学基金基金项目 (61302172) 的阶 段性成果之一。

\section{参考文献}

[1] Pawan Setlur, Moeness Amin, Fauzia Ahmad, et al. Multipath Model and Exploitation in Through-the-Wall and Urban Radar Sensing [J]. IEEE Transactions on Geoscience and Remote Sensing, 2011, Vol.49 (10): 4021-4034.

[2] W Zhang, M G Amin, F. Ahmad, A Hoorfar, and G E Smith, Ultrawideband impulse radar through-the-wall imaging with compressive sensing $[\mathrm{J}]$. International Journal of Antennas and Propagation, 2012, vol. 2012, p. 11.

[3] 赵或, 黄春琳, 栗毅, 雷文太. 超宽带穿墙探测雷达的反向 投影成像算法 $[J]$. 雷达科学与技术, 2007, Vol. 5（1）: 49-54。

[4] D Donoho. Compressed sensing [J]. IEEE Transactions on Information Theory, 2006, Vol. 52(2) : 5406-5425.

[5] Suksmono A B, Bharata E, Lestari A, et al. Compressive stepped-frequency continuous-wave ground penetrating radar [J]. IEEE Geoscience Remote Sensing Letters, 2010, Vol.7(4): 665-669.

[6] 屈乐乐, 方广有, 杨天虹. 压缩感知理论在频率步进探地雷 达偏移成像中的应用 $[\mathrm{J}]$. 电子与信息学报, 2011, 33 (1): 21-26。

[7] Michael Leigsnering, Moeness G Amin and Fauzia Ahmad, Multipath exploitation and suppression for SAR imaging of building interiors [J]. IEEE Signal Processing Magazine, 2014, Vol. 31(4): 110-119.

[8] 吴仁彪, 刘家学, 张蓓. 探地雷达地杂波抑制方法研究进展 [J]. 信号处理, 2005, Vol.21 (4A)：510-513。

[9] Candes E J, Wakin M B, An introduction to compressed sampling [J]. IEEE Signal Processing Magazine, 2008, Vol. 25(2): 21-30. 
[10] D Needell and $\mathrm{R}$ Vershynin, Uniform uncertainty principle and signal recovery via regularized orthogonal matching pursuit $[\mathrm{J}]$. Foundations of Computational Mathematics, 2009, Vol.9(3): 317-334.
[11] C H Seng, A Bouzerdoum, M G Amin and S L Phung, Two-stage fuzzy fusion with applications to through-the-wall radar imaging [J]. IEEE Geoscience and Remote Sensing Letters, 2012, 10, 687-691. 